\title{
Detailing the Structure of Liquid THF Based on an EPSR Study
}

\author{
Alexandre Borges, João M. M. Cordeiro \\ Department of Physics and Chemistry, São Paulo State University, Ilha Solteira, Brazil \\ Email: ${ }^{*}$ cordeiro@dfq.feis.unesp.br
}

Received 22 October 2014; revised 26 November 2014; accepted 24 December 2015

Academic Editor: Mulpuri Nagaraju, Indiana University-Perdue University, USA

Copyright (C) 2015 by authors and Scientific Research Publishing Inc.

This work is licensed under the Creative Commons Attribution International License (CC BY).

http://creativecommons.org/licenses/by/4.0/

cc) (i) Open Access

\begin{abstract}
The structure of pure liquid tetrahydrofuran (THF) has been investigated via Monte Carlo simulations in the wake of a previous Empirical Potential Structure Refinement (EPSR) study on that liquid (Bowron, D.T.; Finney, J.L.; Soper, A.K. (2006) J. Am. Chem. Soc., 128, 5119). The molecules are all-atoms rigid structures and the intermolecular potential used is described for the classical 6-12 Lennard-Jones plus Coulomb in the NPT ensemble at $1 \mathrm{~atm}$ and $25^{\circ} \mathrm{C}$. THF is a poorly structured liquid. Typical preferred orientation of molecules has been explored and calculations shown different types of molecular pairs exist concurrently in the liquid. The geometry of those pairs was deeply investigated and its influence in the liquid structure discussed. The lack of molecular organization in the liquid is closely related to the existence of that diversity of molecular pairs. Its geometry changes from antiparallel up to T-like depending on the distance between the molecules in the pairs.
\end{abstract}

\section{Keywords}

Monte Carlo Simulations, Tetrahydrofuran, Liquid Structure

\section{Introduction}

Tetrahydrofuran (THF) is a small cyclic ether frequently adopted as solvent, thanks to its remarkable physicochemical properties (see Figure 3 for the molecular structure). The molecule of THF is a very interesting model for studies related to structure of organic rings, torsional barriers and vibrational modes. A series of reported results point out that the molecule presents pseudo-rotation, which consists in the transition from the Cs (envelope) to the C2 (twist) conformation [1]-[3] with the last one being the most stable of them [4].

*Corresponding author. 
As a structural unity of many carbohydrates, it is a precursor of biologically active molecules [5]. Lots of important natural substances contain the THF structure in their molecules as, for example, marine macrolides and tetrahydrofuran lignans, just to name two that are subject of study in our laboratory. Marine macrolides are secondary metabolites with important biological activities as a defense mechanism to the aggression of the environment. Because of that they are excellent candidates for the investigation of new bioactive molecules with high pharmacological potential [6] [7]. Some of them have reached the clinical trial stage or the market, as is the case of the anticancer agent eribulin, the analogue of the macrolide halicondrin B [8] [9]. On the other hand, some tetrahydrofuran lignans have showed a broad range of biological activities, including anti-inflammatory, analgesic, trypanocidal, schistosomicidal, antileishmanial and antimalarial [10]-[15]. No less important is that THF molecule can be used as an analogous of the DNA skeleton, since DNA can be viewed as a chain consisting of various THF molecules linked each other by phosphate ions. This model has been used, for example, in studies on DNA mutations induced by ionizing radiation [16].

Liquid THF does not spontaneously produce solvated electrons on solvation of alkali metal atoms such as sodium [17]. It is thought to allow the creation of larger volumes in which free electrons can be accommodated [18] [19]. Because of that, it has recently been used as a novel solvent in investigations of solvated electron that are of importance in improving our understanding of electron-transfer reactions and radiation chemistry [17] [18].

Most of the current understanding of THF liquid structure is almost exclusively derived from computer simulation studies [20]-[24]. All these investigations have been parameterized to reproduce reasonably macroscopic physical properties such as the liquid's diffusion coefficient or specific heat capacity. Because of that the resulting structure is entirely dependent upon the resulting interatomic and intermolecular force fields and conclusions concerning molecular interactions and local structure are largely speculative [25]. Recently, we began a systematic study on the structure of liquids, both pure [26] as mixtures [27] [28], using neutron diffraction with isotope substitution and EPSR simulation [29]-[31]. In a previous application of that methodology Bowron et al. have investigated the structure of liquid THF detailing the molecular correlations and the void structure in the liquid [25]. The present study comes on the heels of those previously cited aiming to deep the knowledge on the structure of liquid THF. For that, the classic Monte Carlo approach used in the EPSR methodology was applied to equilibrate the liquid and the simulation box explored using a protocol developed in our group [32]. The results complement those reported previously and help to have a more detailed view of the structure of the liquid.

\section{Methodology}

\subsection{Molecular Models and Intermolecular Potential Function}

The molecular geometry used for the THF molecule has been that derived previously from neutron diffraction with EPSR methodology [25]. In the present simulations have been used the intermolecular potential optimized for Chandrasekhar and Jorgensen [20] [21]. The molecules are all-atoms represented and were maintained rigid during the simulation which is a quite appropriated approximation for THF, as it has been reported [20] [21]. Of course, this approach introduces uncertainties in the simulations which can be significant if one studies strongly conformer-dependent properties such as molecular polarization for example, which it is not the case here.

As it is classically performed in this kind of simulations, the energy $E_{a b}$ between the interacting sites in the molecules a and $\mathbf{b}$ is calculated by a sum of Coulomb and Lennard Jones potentials centered on the sites:

$$
E_{a b}=\sum_{i j}\left[\frac{A_{i j}}{r_{i j}^{12}}-\frac{B_{i j}}{r_{i j}^{6}}+\frac{q_{i} q_{j}}{r_{i j}}\right]
$$

here, $r_{i j}$ is the distance between site $i$ in $\mathbf{a}$ and site $j$ in $\mathbf{b}$ and $q_{i}$ and $q_{j}$ are fractional charges located on the $i$ and $j$ molecular sites. For each site $k$, the parameters $A_{k k}$ and $B_{k k}$ were given by $A_{k k}=4 \varepsilon_{k} \sigma_{k}^{12}$ and $B_{k k}=4 \varepsilon_{k} \sigma_{k}^{6}$, where $\varepsilon_{k}$ and $\sigma_{k}$ are the Lennard-Jones parameters for the $k$ th site. Parameters $A_{i j}$ and $B_{i j}$ for non-diagonal interactions $[i, j]$ were obtained using the geometric combining rules $A_{i j}=\left(A_{i i} A_{j j}\right)^{1 / 2}$ and $B_{i j}=\left(B_{i i} B_{j j}\right)^{1 / 2}$ [33]. As it is easily realized, this is a pairwise potential where $E_{a b}$ is the energy between the molecular pairs.

\subsection{Monte Carlo Simulations}

The simulations were carried out in the NPT ensemble at $298 \mathrm{~K}$ and $1 \mathrm{~atm}$, with Metropolis importance sampling and periodic boundary conditions [33] on systems consisting of a cubic box containing 500 molecules. In the calculation of the total configurational energy using Equation (1), a full intermolecular interaction was con- 
sidered whenever the $r_{i j}$ site-to-site distance gets inside a cutoff radius of $11 \AA$. Considering that for neutral molecules the main contribution to the cohesive energy comes from the dipole-dipole interaction, which decrease asymptotically with $1 / r^{6}$ [34], long-range interactions beyond the cutoff radius were not considered. The use of approaches like Ewald summation, for example, also very used in our laboratory, does not introduce any gain in the results [35] [36].

\section{Results and Discussion}

It is not the aim of this work to analyze the radial distribution functions g(r) obtained, since they were detailing analyzed previously [25]. However, just to facilitate the discussion and give a first view on the liquid, it is presented in Figure 1 the pair distribution functions for the heavy atoms obtained from the simulations.

It is clear from the curves that THF is a liquid poorly organized. All radial distribution functions started about $3 \AA$ with very broad curves (some correlations with methyl hydrogen atoms are somewhat shorter), reflex of the weak interaction among molecules. Accordingly, Borow et al. reported a coordination number of 12.6 for the distribution of the molecular centers, which is the typical coordination number for the distribution of hard spheres [25]. The interactions between the molecules are structured by the oxygen and the $\mathrm{C}(\mathrm{O})$ sites. Furthermore, the interactions at the non-polar part of the molecule are driven by the general molecular packing within the liquid, as it has been discussed [25]. Typical preferred orientation of molecules has been explored through intermolecular orientational correlation maps (OCM) [25]. The authors shown there are three preferred pairs formed in the liquid, all of them T-like with the central molecule pointing into the center of a neighboring THF molecular ring. However, no information is given on the stability of these pairs. Thus, to have an idea about that stability, the pair energy distribution along the simulation has been accumulated which result is shown in Figure 2 (left)

Differently of other liquids like acetone and N-methylformamide (NMF) [37] or dimethylsulfoxide (DMSO) and water [38], which present an structured pair energy distribution, consequence of existence of pairs with different stability in the liquids, for THF the pair energy distribution curve show no structure. There is a unique band of energy, indicating that the energy relative to the different pairs corresponding to the OCM is encompassed for that band. Accordingly, the three molecular pairs reported are very similar [25]. Furthermore, the range of energy distribution $\left(-3-0 \mathrm{kcal} \cdot \mathrm{mol}^{-1}\right)$ is less negative than that observed for NMF or DMSO, for example [37] [38], indicating weak intermolecular interaction in THF, agreeing with the absence of structure suggested based in the g(r) plots. The mono-modal curve observed in Figure 2 is straight in line with the single peak structure of molecular centers distribution function for THF reported [25]. These results are reflected directly in the absence of any trace of double layer in the liquid, as it is clear from the graph shown in Figure 2 (right).

Hence, from the OCM were derived three preferential T-like molecular pairs [25] which have been showing here are encompassed by a single band of energy. However, although the T-like configurations are the most favorable, the OCM suggest that it is possible for THF molecular rings to interact via antiparallel face to face approach [25]. Having in mind this statement, the simulation box was explored looking for this type of correlation pairs. The search was carried out at well-defined ranges of distance, in order to detail the different types of pair in each region as much as possible. Figure 3 shows three parallel pairs obtained as a function of the interval of distance together with some common geometric parameters.

The results show that, effectively there are parallel pairs in the liquid however they have a tendency to change from completely antiparallel to less antiparallel at larger distances. Concomitantly, as the distance increase, they tend to the T-like form. These are interesting results, since they permit to have a better insight on the behavior of the antiparallel pairs that have been proposed previously [25]. On the other hand, even though the diversity of pairs found in the solvent, the geometric parameters shown are roughly the same for all of them, consequence of the change in the relative position of the molecules. Accordingly, these values rather agree with the distances of the peaks seen in the g(r) plots of Figure 1. Thus, the liquid is effectively constituted of T-like and antiparallel pairs, with the last ones changing from antiparallel to parallel as a function of the distance between the molecules and tending to the T-like for larger distances. Note that this result does not mean that the T-like pairs exist only at larger distances, but that the antiparallel pairs, that are most stable at short distances, tend to change for T-like as the intermolecular distance increase. The presence in the solvent of all these types of molecular pairs may implicate in, or it is a consequence of the poor packing of the THF. 

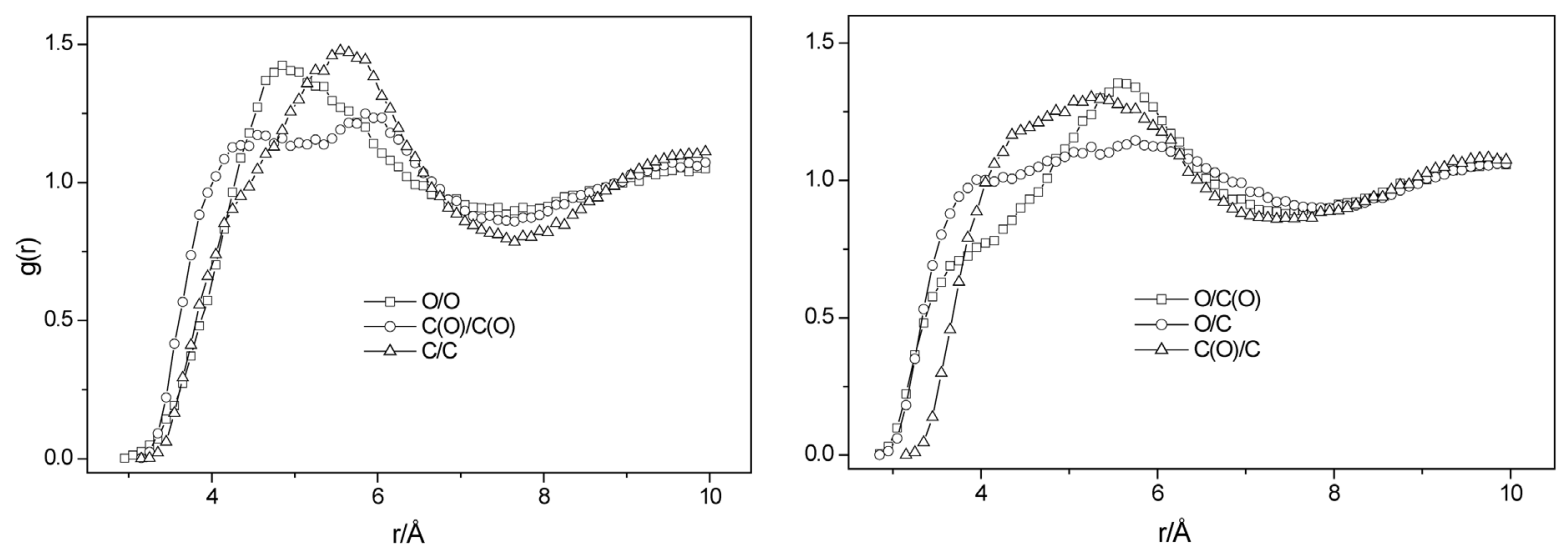

Figure 1. Intermolecular site-site radial distribution functions derived from the simulations for THF at room temperature. $\mathrm{O}$ = oxygen, $\mathrm{C}(\mathrm{O})$ = carbon bonded to oxygen, $\mathrm{C}$ = carbon bonded to $\mathrm{C}(\mathrm{O})$.
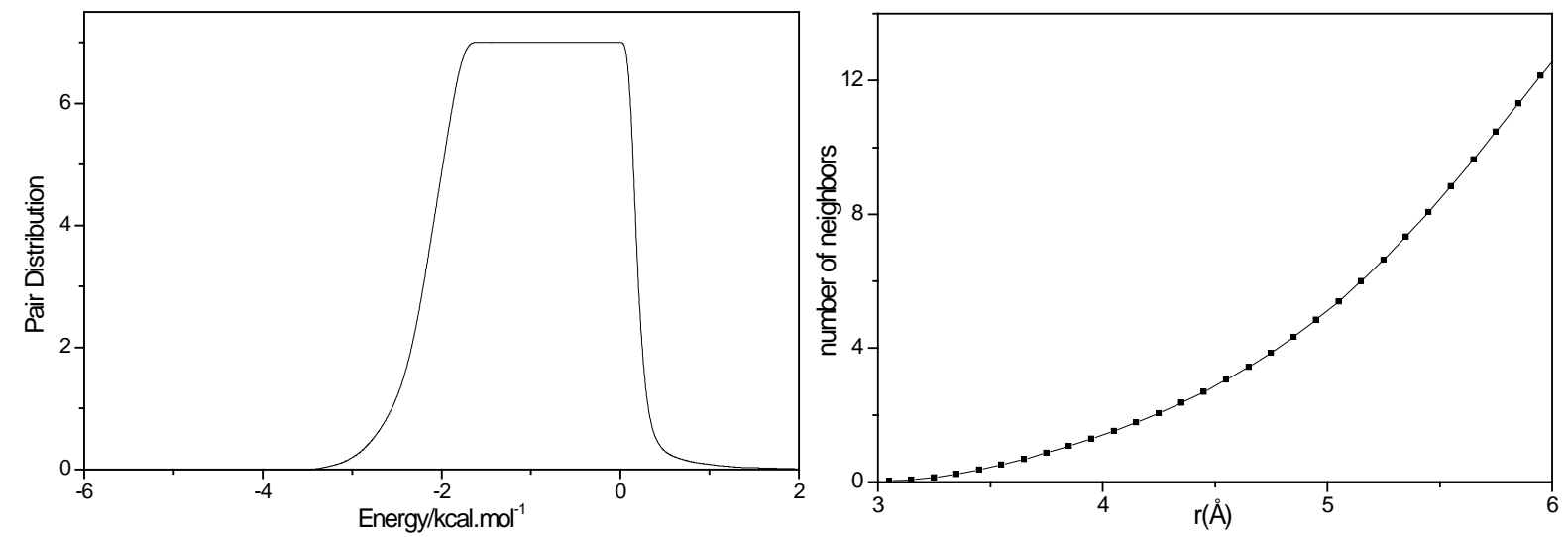

Figure 2. Pair energy distributions derived by the simulation for THF molecules in the liquid (the curves were truncated at 7 for plotting) (left); Number of neighbors as a function of distance between the molecules (right).

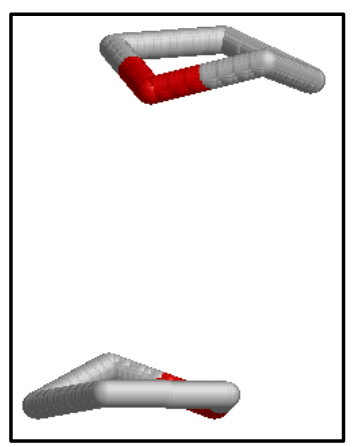

(a)

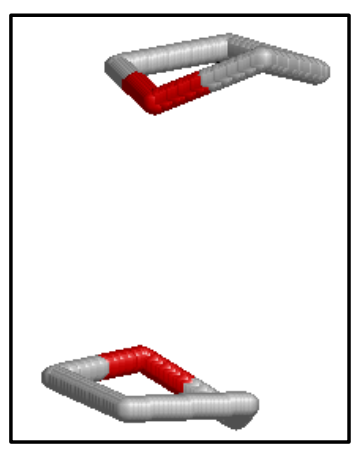

(b)

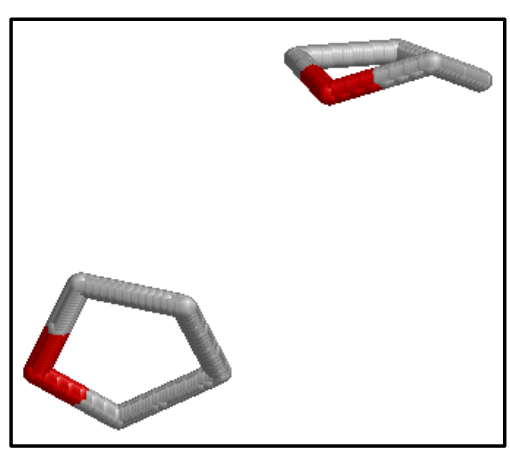

(c)

Figure 3. Parallel pairs as a function of the intermolecular distance in the liquid. (a) 3 - $4 \AA$; (b) 4 $6 \AA$; (c) 6 - $7.5 \AA$. Some geometric parameters common to the pairs: $\mathrm{r}(\mathrm{O} \cdots \mathrm{C}(\mathrm{O}))=3.53 \AA$, $\mathrm{r}(\mathrm{O} \cdots \mathrm{O})$

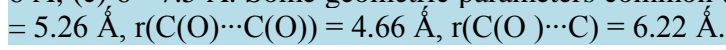

The relative positioning of molecules in the pairs may be even clearer, observing the relative positioning of the dipole moment vectors as a function of the distance, result that is shown in Figure 4. It is clear from the graphic that the molecules in the pairs are antiparallel to molecular distances between 3 and $4 \AA$, with the angle between the dipole moment vectors about $130^{\circ}$ and that the angles tend to values about $90^{\circ}$ for larger molecular distances. These results agree quite well with the whole molecular analyses discussed here and previously as well [25]. 


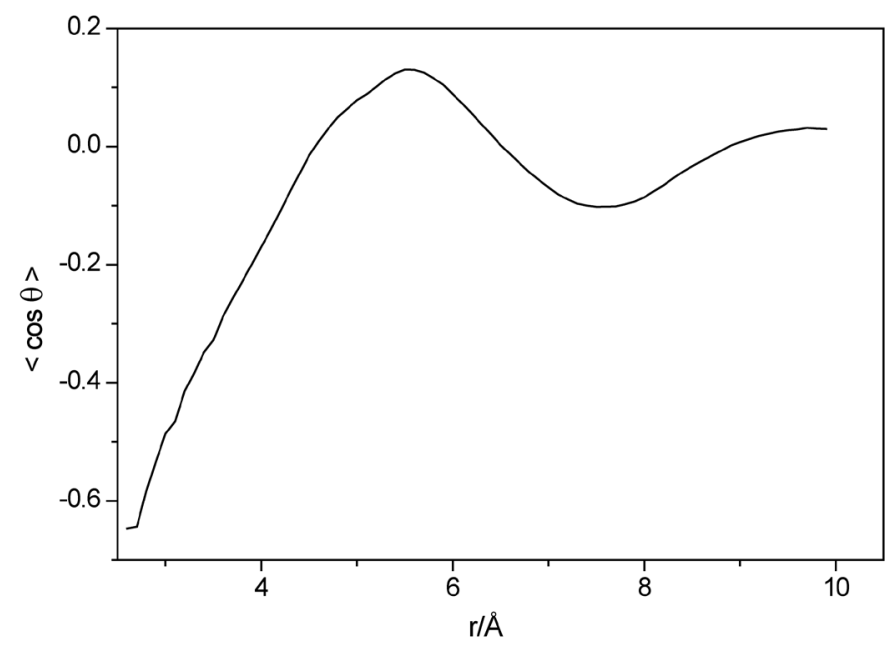

Figure 4. Average dipole-dipole correlation as a function of oxygenoxygen distance. $\theta$ is the angle between the dipole moments of the two THF molecules considered.

\section{Conclusion}

Liquid THF is a popular solvent, thanks to its remarkable physicochemical properties, besides being a structural unity of many important natural substances. It is also thought to allow the creation of larger volumes in which free electrons can be accommodated. Because of that, it has recently been used as a novel solvent in investigations of solvated electrons. The structure of the liquid has been explored via Monte Carlo simulations in the NTP ensemble having a previous EPSR study as starting point. The results point to the existence of different types of pairs, changing from antiparallel to T-shape, depending on the distance between the molecules. It is suggested that this diversity of pairs is closely related with the absence of packing and the formation of voids in the liquid.

\section{Acknowledgements}

A.B. thanks CAPES (Coordenação de Aperfeiçoamento de Pessoal de Nível Superior) for the concession of a grant that help supporting this work.

\section{References}

[1] Cadioli, B., Gallinella, E., Coulombeau, C., Jobic, H. and Berthier, G. (1993) Geometric Structure and Vibrational Spectrum of Tetrahydrofuran. The Journal of Physical Chemistry, 97, 7844-7856. http://dx.doi.org/10.1021/j100132a010

[2] Engerholm, G.G., Luntz, A.C. and Gwinn, W.D. (1969) Ring Puckering in Five-Membered Rings. II. The Microwave Spectrum, Dipole Moment, and Barrier to Pseudorotation in Tetrahydrofuran. The Journal of Physical Chemistry, 50, 2446. http://dx.doi.org/10.1063/1.1671401

[3] Greenhouse, J. and Strauss, H.L. (1969) Spectroscopic Evidence for Pseudorotation. II. The Far-Infrared Spectra of Tetrahydrofuran and 1,3-Dioxolane. The Journal of Chemical Physics, 50, 124. http://dx.doi.org/10.1063/1.1670769

[4] Rosas, R.L., Cooper, C. and Laane, J. (1990) Evaluation of Molecular Mechanics Methods for the Calculation of the Barriers to Planarity and Pseudorotation of Small Ring Molecules. The Journal of Physical Chemistry, 94, 1830-1836. http://dx.doi.org/10.1021/j100368a021

[5] Werawattanachai, N., Towiwat, P., Unchern, S. and Maher, T.J. (2007) Neuropharmacological Profile of Tetrahydrofuran in Mice. Life Sciences, 80, 1656-1663. http://dx.doi.org/10.1016/j.lfs.2007.01.050

[6] Bhatnagar, I. and Kim, S.K. (2010) Marine Antitumor Drugs: Status, Shortfalls and Strategies. Marine Drugs, 8, $2702-$ 2720. http://dx.doi.org/10.3390/md8102702

[7] Lorente, A., Lamariano-Merketegi, J., Albericio, F. and Álvarez, M. (2013) Tetrahydrofuran Containing Macrolides: A Fascinating Gift from the Deep Sea. Chemical Reviews, 113, 4567-4610.

[8] Jackson, K.L., Henderson, J.A. and Phillips, A.J. (2009) The Halichondrins and E7389. Chemical Reviews, 109, 30443079. http://dx.doi.org/10.1021/cr900016w 
[9] Ledford, H. (2010) Complex Synthesis Yields Breast-Cancer Therapy. Nature, 468, 608-609. http://dx.doi.org/10.1038/468608a

[10] Ren, Y., Chai, H., Goetz, M. and Kinghorn, A.D. (2013) A Cytotoxic Decahydronaphthalenylpropenal Derivative and Tetrahydrofuran Lignans from the Stems of Cameraria latifolia. Tetrahedron Letters, 54, 4854-4858. http://dx.doi.org/10.1016/j.tetlet.2013.06.109

[11] Chi, N.-J., Liu, Y. and Aisa, H.A. (2012) Tetrahydrofuran Lignans and Flavonoids from Artemisia absinthium. Chemistry of Natural Compounds, 48, 666-667. http://dx.doi.org/10.1007/s10600-012-0342-X

[12] Silva-Filho, A.A., Costa, E.S., Cunha, W.R., Silva, M.L.A., Nanayakkara, N.P.D. and Bastos, J.K. (2008) In Vitro Antileishmanial and Antimalarial Activities of Tetrahydrofuran Lignans Isolated from Nectandra megapotamica (Lauraceae). Phytotherapy Research, 22, 1307-1310. http://dx.doi.org/10.1002/ptr.2486

[13] Royo, V.A., Santos, F.F., Souza, V.A., Pereira, A.C., Silva, R., Vinhólis, A.H.C., Donate, P.M., Silva, M.L.A., Albuquerque, S. and Bastos, J.K. (2003) Biological Activity Evaluation of Dibenzylbutyrolactone Lignans Derivatives against Leishmania braziliensis. Brazilian Journal of Pharmacognosy, 13, 18-21.

[14] Souza, V.A., Silva, R., Pereira, A.C., Royo, V.D., Saraiva, J., Montanheiro, M., Souza, G.H.B., Silva, A.A., Grando, M.D., Donate, P.M., Bastos, J.K., Albuquerque, S. and Silva, M.L.A.E. (2005) Trypanocidal Activity of (-)-Cubebin Derivatives against Free Amastigote forms of Trypanosoma cruzi. Bioorganic \& Medicinal Chemistry Letters, 15, 303307. http://dx.doi.org/10.1016/j.bmcl.2004.10.079

[15] Neto, A.G., Silva, A.A., Costa, J.M.L.C., Vinholis, A.H.C., Souza, G.H.B., Cunha, W.R., Silva, M.L.A.E., Albuquerque, S. and Bastos, J.K. (2004) Evaluation of the Trypanocidal and Leishmanicidal in Vitro Activity of the Crude Hydroalcoholic Extract of Pfaffia glomerata (Amarathanceae) Roots. Phytomedicine, 11, 662-665. http://dx.doi.org/10.1016/j.phymed.2003.06.005

[16] Lepage, M., Letarte, S., Michaud, M., Motte-Tollet, F., Hubin-Franskin, M.J., Roy, D. and Sanche, L. (1998) Electron Spectroscopy of Resonance-Enhanced Vibrational Excitations of Gaseous and Solid Tetrahydrofuran. The Journal of Chemical Physics, 109, 5980. http://dx.doi.org/10.1063/1.477223

[17] Barthel, E.R., Martini, I.B. and Schwartz, B.J. (2000) Direct Observation of Charge-Transfer-to-Solvent (CTTS) Reactions: Ultrafast Dynamics of the Photoexcited Alkali Metal Anion Sodide ( $\mathrm{Na}^{-}$). The Journal of Chemical Physics, 112, 9433. http://dx.doi.org/10.1063/1.481563

[18] Martini, I.B., Barthel, E.R. and Schwartz, B.J. (200) Mechanisms of the Ultrafast Production and Recombination of Solvated Electrons in Weakly Polar Fluids: Comparison of Multiphoton Ionization and Detachment via the ChargeTransfer-to-Solvent Transition of $\mathrm{Na}^{-}$in THF. The Journal of Chemical Physics, 113, 11245. http://dx.doi.org/10.1063/1.1328071

[19] Bedard-Hearn, M.J., Larsen, R.E. and Schwartz, B.J. (2005) The Role of Solvent Structure in the Absorption Spectrum of Solvated Electrons: Mixed Quantum/Classical Simulations in Tetrahydrofuran. The Journal of Chemical Physics, 122, Article ID: 134506. http://dx.doi.org/10.1063/1.1867378

[20] Chandrasekhar, J. and Jorgensen, W.L. (1982) Monte Carlo Simulations of Liquid Tetrahydrofuran including Pseudorotation. The Journal of Chemical Physics, 77, 5073. http://dx.doi.org/10.1063/1.443681

[21] Chandrasekhar, J. and Jorgensen, W.L. (1982) The Nature of Dilute Solutions of Sodium Ion in Water, Methanol, and Tetrahydrofuran. The Journal of Chemical Physics, 77, 5080. http://dx.doi.org/10.1063/1.443682

[22] Bedard-Hearn, M.J., Larsen, R.E. and Schwartz, B.J. (2003) Understanding Nonequilibrium Solute and Solvent Motions through Molecular Projections: Computer Simulations of Solvation Dynamics in Liquid Tetrahydrofuran (THF). The Journal of Physical Chemistry B, 107, 14464-14475. http://dx.doi.org/10.1021/jp035846e

[23] Girard, S. and Muller-Plathe, F. (2003) Molecular Dynamics Simulation of Liquid Tetrahydrofuran: On the Uniqueness of Force Fields. Molecular Physics, 101, 779-787. http://dx.doi.org/10.1080/0026897021000054817

[24] Rayon, V.M. and Sordo, J.A. (2005) Pseudorotation Motion in Tetrahydrofuran: An Ab Initio Study. The Journal of Chemical Physics, 122, Article ID: 204303. http://dx.doi.org/10.1063/1.1899123

[25] Bowron, D.T., Finney, J.L. and Soper, A.K. (2006) The Structure of Liquid Tetrahydrofuran. Journal of the American Chemical Society, 128, 5119-5126. http://dx.doi.org/10.1021/ja0583057

[26] Cordeiro, J.M.M. and Soper, A.K. (2009) Neutron Diffraction Study of Liquid N-Methylformamide Using EPSR Simulation. The Journal of Physical Chemistry B, 113, 6819-6825. http://dx.doi.org/10.1021/jp902053y

[27] Cordeiro, J.M.M. and Soper, A.K. (2011) Investigation on the Structure of Liquid N-Methylformamide-Dimethylsulfoxide Mixtures. Chemical Physics, 381, 21-28. http://dx.doi.org/10.1016/j.chemphys.2011.01.003

[28] Cordeiro, J.M.M. and Soper, A.K. (2013) A Hybrid Neutron Diffraction and Computer Simulation Study on the Solvation of $N$-Methylformamide in Dimethylsulfoxide. The Journal of Chemical Physics, 138, Article ID: 044502. http://dx.doi.org/10.1063/1.4773346 
[29] Finney, J.L. and Soper, A.K. (1994) Solvent Structure and Perturbations in Solutions of Chemical and Biological Importance. Chemical Society Reviews, 23, 1-10. http://dx.doi.org/10.1039/cs9942300001

[30] Soper, A.K. (1996) Empirical Potential Monte Carlo Simulation of Fluid Structure. Chemical Physics, 202, $295-306$. http://dx.doi.org/10.1016/0301-0104(95)00357-6

[31] Soper, A.K. (1996) Partial Structure Factors from Disordered Materials Diffraction Data: An Approach Using Empirical Potential Structure Refinement. Physical Review B, 72, Article ID: 104204. http://dx.doi.org/10.1103/PhysRevB.72.104204

[32] Freitas, L.C.G. (2009) DIADORIM: A Monte Carlo Program for Liquid Simulations including Quantum Mechanics and Molecular Mechanics (QM/MM) Facilities: Applications to Liquid Ethanol. Journal of the Brazilian Chemical Society, 20, 1541-1548. http://dx.doi.org/10.1590/S0103-50532009000800022

[33] Allen, M. and Tildesley, D.J. (1987) Computer Simulation of Liquids. Oxford University Press, Oxford.

[34] Tabor, D. (1968) Gases, Liquids and Solids. Penguin Library of Physical Sciences, Cambridge.

[35] Cordeiro, J.M.M. (2007) Structure of Acetone and Dimethyl Sulfoxide from Monte Carlo Simulations and MM2 Calculations. Physics and Chemistry of Liquids, 45, 31-39. http://dx.doi.org/10.1080/00319100600941748

[36] Cordeiro, J.M.M. and Bosso, A.R.S.A. (2010) Monte Carlo Studies of N-Methylformamide-Dimethyl Sulfoxide Mixtures. Journal of Molecular Liquids, 154, 36-40. http://dx.doi.org/10.1016/j.molliq.2010.03.006

[37] Almeida, G.G. and Cordeiro, J.M.M. (2011) A Monte Carlo Revisiting of N-Methylformamide and Acetone. Journal of the Brazilian Chemical Society, 22, 2178-2185. http://dx.doi.org/10.1590/S0103-50532011001100022

[38] Borges, A. and Cordeiro, J.M.M. (2013) Hydrogen Bonding Donation of $N$-Methylformamide with Dimethylsulfoxide and Water. Chemical Physics Letters, 565, 40-44. http://dx.doi.org/10.1016/j.cplett.2013.02.017 
Scientific Research Publishing (SCIRP) is one of the largest Open Access journal publishers. It is currently publishing more than 200 open access, online, peer-reviewed journals covering a wide range of academic disciplines. SCIRP serves the worldwide academic communities and contributes to the progress and application of science with its publication.

Other selected journals from SCIRP are listed as below. Submit your manuscript to us via either submit@scirp.org or Online Submission Portal.
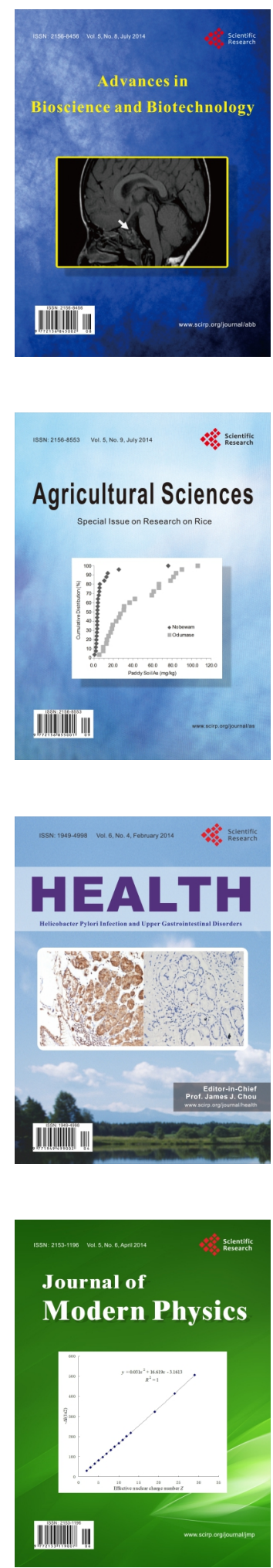
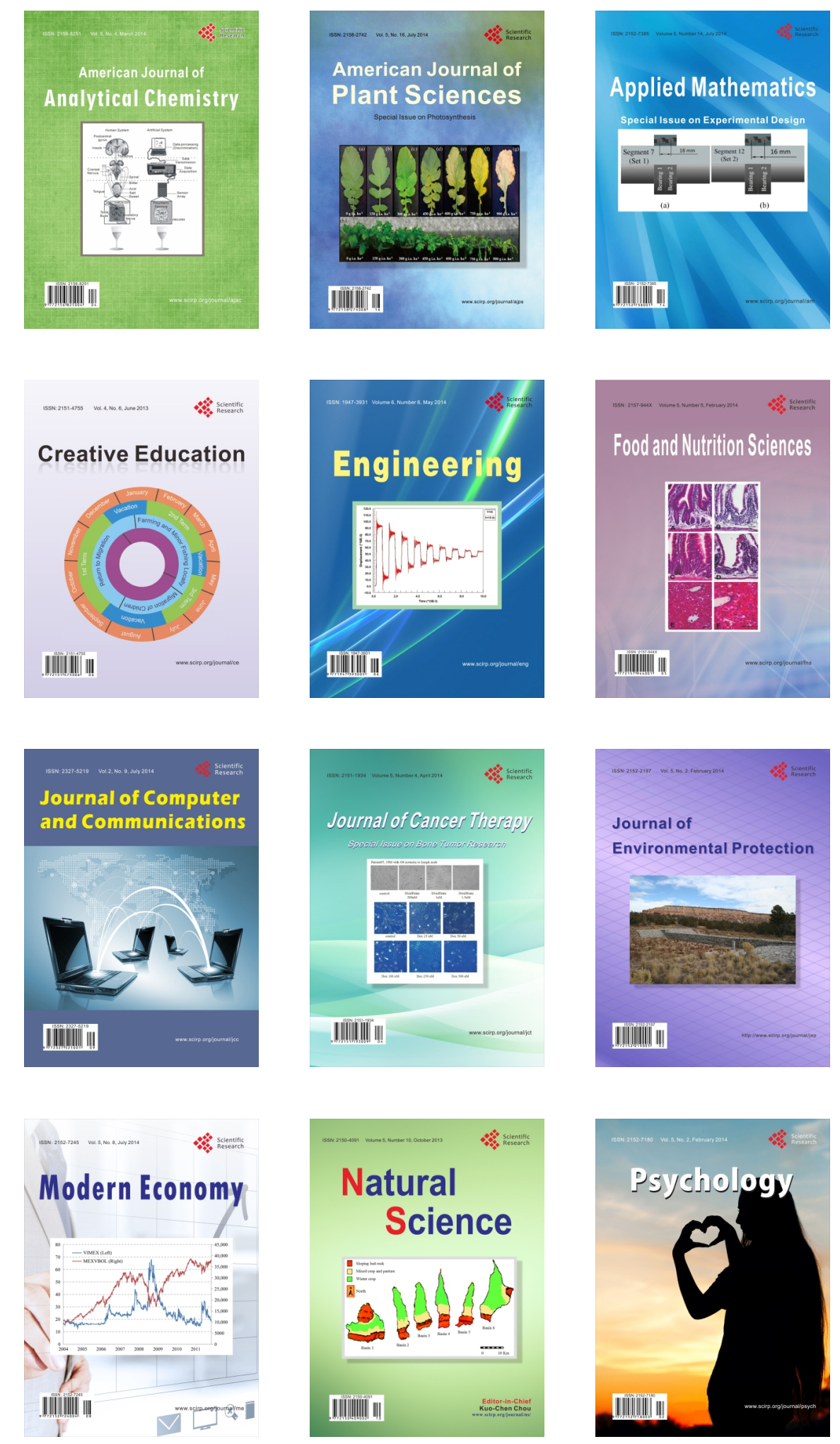